\title{
Fast processes in semiconductor optical amplifiers: theory and experiment
}

\author{
Mørk, Jesper
}

Published in:

2002 Digest of the LEOS Summer Topical Meetings

Link to article, DOI:

10.1109/LEOSST.2002.1027582

Publication date:

2002

Document Version

Publisher's PDF, also known as Version of record

Link back to DTU Orbit

Citation (APA):

Mørk, J. (2002). Fast processes in semiconductor optical amplifiers: theory and experiment. In 2002 Digest of the LEOS Summer Topical Meetings IEEE Press. https://doi.org/10.1109/LEOSST.2002.1027582

\section{General rights}

Copyright and moral rights for the publications made accessible in the public portal are retained by the authors and/or other copyright owners and it is a condition of accessing publications that users recognise and abide by the legal requirements associated with these rights.

- Users may download and print one copy of any publication from the public portal for the purpose of private study or research.

- You may not further distribute the material or use it for any profit-making activity or commercial gain

- You may freely distribute the URL identifying the publication in the public portal

If you believe that this document breaches copyright please contact us providing details, and we will remove access to the work immediately and investigate your claim. 
TuK3'(Invited)

2:30 pm-3:00 pm

\title{
Fast Processes in Semiconductor Optical Amplifiers: Theory and Experiment
}

\author{
Jesper Mørk \\ COM, Technical University of Denmark \\ Building 345V, DK-2800 Kgs. Lyngby, Denmark \\ E-mail: jm@com.dtu.dk, Tel: +45 4525 5765, Fax: +45 45936581
}

\begin{abstract}
We review the physical processes responsible for ultrafast gain and index dynamics in semiconductor optical amplifiers and discuss their impact on optical switching applications.
\end{abstract}

All-optical signal processing is expected to play an important role in future high-capacity optical communication systems. Primary incentives for this evolution are the larger data rates that can be handled by all-optical devices, as well as the cost-reduction and increased flexibility that may be achieved by avoiding conversions between the optical and the electronic domain. In order to be practical and competitive, all-optical switching devices should fulfil criteria similar to those of electronics, i.e., be small and allow integration of different functionalities, and have the potential for cheap mass-production. Presently, semiconductor optical amplifier (SOA) based devices are among the primary contenders for integrated all-optical devices. The large gain and large differential gain of SOAs allow switching with power levels in the range of milliwats, and various functionalities have been demonstrated in a number of different schemes at speeds in excess of $100 \mathrm{~Gb} / \mathrm{s}$, e.g. wavelength conversion at $168 \mathrm{~Gb} / \mathrm{s}$ [1]. In this paper we will review and discuss the physical processes that impact the operation of SOAs at such high data-rates.

Common to the various schemes utilizing SOAs as the main switching element is the exploitation of saturation effects in the active region of the waveguide. When an optical (pump) beam is injected in the amplifier, the gain of the amplifier is saturated and, in consequence of the induced change of the carrier density in the active region, the refractive index of the waveguide is changed. Both effects can be utilized for all-optical switching of a data signal. In the simplest scheme of cross-gain modulation (XGM), the gain change simply controls the amplitude of another (probe) beam transmitted through the waveguide. The refractive index change is exploited in interferometer structures, where the cross-phase modulation (XPM) imparted on the probe beam in one arm of an interferometer can be converted to an amplitude change.

Following depletion by an injected optical pulse, the gain of the SOA will recover to its initial value by processes that restore the carrier distribution in the active region. The gain and the index of the device in general depend on the detailed distribution of carriers in energy, but can be parameterised by the carrier density, if a quasi-equilibrium situation can be assumed. The recovery of the carrier density in the active region is governed by the so-called stimulated carrier lifetime, i.e., the lifetime decreases when the optical power level is high. This can be utilized to increase the bandwidth of the SOA by ensuring a high optical power level, either through the data/control beams themselves and an amplifier with high gain or by the application of a separate "holding" beam [2]. In this way gain recovery times as low as a few tens of picoseconds have been realized and this is a main reason for the successful high-speed demonstrations of XGMbased schemes. A related phenomenon is the "self-filtering" of the optical signal that takes place upon propagation in the amplifier [3]. Thus, a modulation component, once generated, has a highpass transmission characteristic through the amplifier, since higher frequencies saturate the - amplifier less. This tends to equalize the lower efficiency with which the high-frequency components can be generated by XGM, thus increasing the bandwidth of the device [3].

Switching windows not limited in width by the carrier lifetime can be realized in interferometers by utilizing that only the phase difference between the two arms matters (differential scheme), thus enabling cancellation of a slow index recovery. Sub-picosecond 
switching windows have been achieved for a periodic control signal [4], but since the magnitude of the index change depends on the saturation of the amplifier, patterning effects anyway limit the bit-rate for data-controlled functionalities, such as wavelength conversion and regeneration.

If the characteristic time scale of the pump signal becomes comparable to or shorter than the sub-picosecond scattering times on which a quasi-equilibrium carrier distribution is established in the device, the detailed evolution of the carrier distribution becomes important. Thus, spectral holeburning and carrier heating effects significantly change the gain and index dynamics for short optical pulses and for high data rates. These effects are well-known to cause gain suppression and bandwidth limitations in semiconductor lasers. The temporal characteristics of the processes as well as the dependence on operation parameters, such as wavelength and injection current, can be characterized through heterodyne pump-probe measurements employing femtosecond optical pulses [5]. A generalized rate equation model has been established that well accounts for such measured gain and index dynamics in bulk and quantum well SOAs [6]. On the 100 fsec time scale, instantaneous (coherent) processes such as two-photon absorption and optical Kerr effects are also found to significantly influence the response.

The ultrafast contributions to the gain response strongly modify the short pulse saturation properties of amplifiers. It has thus been shown, experimentally [7] as well as theoretically [8], that for relatively low repetition rates and pulses shorter than about $10 \mathrm{ps,} \mathrm{ultrafast} \mathrm{gain} \mathrm{dynamics}$ provide the dominant contribution to the saturation of the pulse gain. For optical switches, this has the implication that the carrier density change induced by a pump pulse with fixed energy is reduced for shorter pulses [4,9]. On the other hand, the contributions to the gain and index change from the intraband dynamics increase for shorter pulses [4,5]; leading to switching windows with characteristics that depend on the pulsewidth and the operation point of the device. In the talk we will discuss the contributions of the different physical processes that make up the switching windows, as well as the scaling with material and device parameters.

Lasers with quantum dot (QD) active material have shown record-low threshold current densities and it is of interest to explore the potential of QD SOAs for ultrafast processing [10]. We will present modelling results for QD SOAs, indicating that in this material strong spectral holeburning dynamics may facilitate operation in a new regime [11], where patterning effects due to slow carrier recovery can be reduced below the limits of bulk and quantum well SOAs.

1. S. Nakamura, $Y$. Ueno and $K$. Tajima, " $168-\mathrm{Gb} / \mathrm{s}$ all-optical wavelength conversion with a symmetric-MachZehnder-type switch”, IEEE Photon. Technol. Lett., vol. 13, pp. 1091-1093, Oct. 2001.

2. R. J. Manning, A. D. Ellis, A. J. Poustie, and K. J. Blow, "Semiconductor laser amplifiers for ultrafast all-optical signal processing", J. Opt. Soc. Am. B, vol. 14, pp. 3204-3216, Nov. 1997.

3. C. Jorgensen et al., "All-optical wavelength conversion at bit rates above $10 \mathrm{~Gb} / \mathrm{s}$ using semiconductor optical amplifiers",IEEE J. Select. Topics Quantum Electron., vol. 3, pp. 1168-1180, Oct. 1997.

4. S. Nakamura, Y. Ueno, and K. Tajima, "Femtosecond switching with semiconductor-optical-amplifier-based symmetric Mach-Zehnder-type all-optical switch", App. Phys. Lett., vol 78, pp. 3929-3931, June 2001.

5. K. L. Hall et al., "Nonlinearities in active media", in Nonlinear Optics in Semiconductors II. Semiconductors and Semimetals, vol. 59, Ed. by E. Garmire and A. Kost, Academic Press (San Diego), 1999.

6. J. Mørk and A. Mecozzi, "Theory of the ultrafast optical response of active semiconductor waveguides", J. Opt. Soc. Am. B., vol. 13, pp. 1803-1816, Aug. 1996.

7. P. Borri et al., "Measurement and calculation of the critical pulsewidth for gain saturation in semiconductor optical amplifiers", Opt. Commun., vol. 164, pp. 51-55, June 1999.

8. A. Mecozzi and J. Mørk, "Saturation effects in non-degenerate four-wave mixing between short optical pulses in semiconductor laser amplifiers", IEEE J. Sel.. Topics Quantum Electron., vol. 3, pp. 1190, 1997.

9. R. Schreieck et al., "Ultrafast switching dynamics of Mach-Zehnder interferometer switches", IEEE Photon. Technol. Lett., vol. 13, pp. 603-605, June 2001.

10. M. Sugawara et al., "Quantum-dot semiconductor optical amplifiers for high bit-rate signal processing over 40 Gbit/s", Jpn. J. Appl. Phys., vol. 40, pp. L488-L491, May 2001.

11. T.W. Berg, A.V. Uskov, and J. Mørk, "Ultrafast signal processing in quantum dot amplifiers through effective spectral holeburning", to be presented at CLEO'02, Long Beach, California, May 2002. 